\title{
Effect of Cilostazol-Loaded PCL/PEG Nanocapsules on Abdominal Aortic Tunics and Lipid Profile of Wistar Rats
}

Luís Daniel Silva Pilatti ${ }^{1}$

https://orcid.org/0000-0001-6234-2952

\section{Ricardo Rodrigues ${ }^{1}$}

https://orcid.org/0000-0001-8119-9530

Núbia da Silva Nascimento ${ }^{1}$

https://orcid.org/0000-0002-4578-3008

\author{
Sandra Maria Warumby Zanin ${ }^{2}$ \\ https://orcid.org/0000-0003-1978-4653
}

\author{
Paulo Vitor Farago ${ }^{3}$ \\ https://orcid.org/0000-0002-9934-4027 \\ César Roberto Busato ${ }^{1}$ \\ https://orcid.org/0000-0003-0667-8157
}

\section{Mona Lisa Simionatto Gomes ${ }^{3}$ \\ https://orcid.org/0000-0001-5179-3985}

${ }^{1}$ State University of Ponta Grossa, Department of Medicine, Ponta Grossa, Paraná, Brazil; ${ }^{2}$ Federal University of Paraná, Department of Pharmacy, Curitiba, Paraná, Brazil; ${ }^{3}$ State University of Ponta Grossa, Department of Pharmaceutical Sciences, Ponta Grossa, Paraná, Brazil.

Received: 2020.02.03; Accepted: 2020.02.26.

*Correspondence: luisdanielsp@gmail.com; Tel.: +55-42-998046671 (F.L.).

\section{HIGHLIGHTS}

- Cilostazol-loaded nanocapsules provided no effect on abdominal aortic tunics.

- Cilostazol-loaded nanocapsules improved HLD-c value as well as pure drug.

- Cilostazol-loaded nanocapsules showed the lowest value for triglycerides.

Abstract: Cilostazol (CLZ) is a phosphodiesterase III inhibitor with antiplatelet and vasodilator properties. It has been recently verified that CLZ plays a significant role in the arteries by inhibiting the proliferation and growth of muscle cells, increasing the release of nitric oxide by the endothelium and promoting angiogenesis. Considering these promising effects, the use of nanocapsules may be an interesting strategy to optimize its pharmacokinetics and pharmacodynamics at the vascular level for preventing atherosclerosis. The aim of this study was to evaluate the effect of cilostazol-loaded nanocapsules in the abdominal aortic tunics and on the lipid profile of Wistar rats in order to investigate its potential role in the prevention of atherosclerosis. Thirty-two animals were divided into four groups of eight animals, with 30-day treatment. Group 1 received nanoencapsulated CLZ; Group 2, control nanocapsules with no drug; Group 3, propylene glycol and water; and Group 4, a solution of CLZ in propylene glycol and water. After 30 days, there was no statistically significant difference between the groups regarding the cellularity and thickness of the arterial tunics of the abdominal aorta. However, the group that received nanoencapsulated CLZ (Group 1) had an improvement in HDL-c and triglyceride values compared to unloaded nanocapsules (Group 2).

Keywords: drug carriers; peripheral arterial disease; phosphodiesterase inhibitors; nanocapsules. 


\section{INTRODUCTION}

Peripheral arterial occlusive disease (PAOD) is a chronic pathological process with an estimated prevalence around 15 to $20 \%$ of the patients over 65 years-old, which results from an arterial obstruction secondary to systemic atherosclerotic events $[1,2]$. It occurs more oftenly in hemodynamically vulnerable sites, such as bifurcations and arterial bends. The change from the blood laminar flow to turbulent flow at these sites causes endothelial stress that culminates with endothelial vasodilation and chemokines secretion. These events favor the entry of low-density lipoproteins (LDL-C) and its subsequent oxidation in the subendothelial arterial layer, with consequent local proliferation of muscle cells and fibrous tissue. The association of this proliferation with the development of the atheroma plaque causes the chronic obstruction that characterizes PAOD [3,4].

Aortoiliac Occlusive Disease (AOD) is the PAOD that develops in the aorta and iliac arteries [5,6], characterized mainly by intermittent claudication, and may be associated with erectile dysfunction and a reduction of the peripheral pulse amplitude [7]. The diagnosis is based on clinical presentation and confirmed by imaging (angiotomography, magnetic resonance imaging, ultrasonography) $[6,8]$. In 2007, a document (TASC II) was drawn which classifies AOD into four types (A, B, C, D), depending on the site and degree of arterial stenosis [9]. Its treatment involves clinical measures, associated or not with specific surgical therapies, such as endovascular therapy or surgical revascularization - that may be applied to the most severe subtypes (cases of critical ischemia) of the disease $[5,9]$.

The clinical treatment involves the management of risk factors (smoking, diabetes, hypertension, hyperlipidemia), supervised exercise, medication (antiplatelet drugs, statins, and angiotensin-converting enzyme inhibitors) to reduce the risk of cardiovascular events [6,10-13], since all forms of PAOD may be interpreted as markers of generalized systemic atherosclerosis, a predictive factor for ischemic events, such as stroke and myocardial infarction [1].

In this context, some drugs have shown to be effective in improving intermittent claudication and painfree walking distance [6,11-14]. An example is Cilostazol (CLZ), a phosphodiesterase III (FDE3) inhibitor. The drug acts by decreasing calcium intracellularly by increasing cAMP levels [15-17].

In addition to being a platelet antiaggregant [15-17], it has been found out that this drug inhibits the proliferation and growth of smooth muscle cells of arteries by regulating the E2F transcription factor [18-20]. Studies have also shown that cilostazol increases the release of nitric oxide by the endothelium, by activation of the cAMP/protein kinase A (PKA) system, resulting in vasodilation [21]; as well as promotion of angiogenesis by serum increase of vascular endothelial growth factor (VEGF) [22]. Therefore, cilostazol has antiplatelet, antiproliferative, and vasodilatory properties.

In vivo studies indicate that cilostazol reduces serum triglyceride levels and increases the high-density lipoprotein (HDL-c), an effect that appears to be mediated by increased serum lipoprotein lipase (LPL) levels; but does not have significant effects on low-density lipoprotein (LDL-c) [23,24].

However, the therapeutic regimen may be impaired by the side effects of the drug - headache, diarrhea, abdominal pain, tachycardia, nausea, peripheral edema - due to administration in two daily doses of $100 \mathrm{mg}$ [15]. In addition, it is contraindicated in cases of heart failure (HF), since it has positive chronotropic effects, and a higher morbimortality in patients medicated with PDE3 inhibitors [15,23].

Due to the large number of side effects and its contraindication to heart disease, there is an interest in optimizing its pharmacokinetic and pharmacodynamic. Accordingly, the use of polymer nanocapsules can be an effective strategy, since these nanomeric therapeutic systems can increase the bioavailability, promote prolonged release and direct its distribution [26-30]. Therefore, this study had aimed to evaluate the pharmacological effect of nanoencapsulated cilostazol on abdominal aortic artery tunics (thickness and cellularity) and on the lipid profile of healthy Wistar rats, studying the impact of this formulation in the proliferation of muscle cells and in its possible therapeutic use in PAOD.

\section{MATERIAL AND METHODS}

Pure cilostazol was purchased from IPCA Laboratories Limited, Mumbai, India, with 99.91\% purity. Poly( $\varepsilon$-caprolactone) (PCL, $M w=10,000-14,000 \mathrm{~g} / \mathrm{mol}$, Sigma-Aldrich, St. Louis, MO, USA) and poly(ethylene glycol) (PEG, Mw= 5400-6600 g/mol, Cromato Produtos Químicos, Diadema, Brazil) were used as received. The cilostazol-loaded PCL-PEG nanocapsules at $12 \mathrm{mg} / \mathrm{mL}$ were obtained by the interfacial deposition of preformed polymer method as described by Gomes and coauthors. [28]. This formulation was previously characterized by particle size, zeta potential, encapsulation efficiency, X-ray diffraction and infrared spectroscopy [28], with the purpose of assuring the quality requirements for the proposed use. The propylene glycol solvent was also acquired from Sigma-Aldrich (St. Louis, MO, USA) and 
used as received. Ultrapurified water was obtained by Milli-Q® reverse osmosis system (Millipore, Bedford, MA, USA).

For the in vivo assay, 32 healthy, 60-day-old male Wistar rats (Rattus norvergicus) were kept in cages containing four animals each and maintained at an environment with temperature $\left(22 \pm 2^{\circ} \mathrm{C}\right)$ and light-dark cycle of $12 / 12 \mathrm{~h}$ (automatically controlled), water and commercial feed (Nuvilab ${ }^{\circledR}$ ) ad libidum. The experimental protocol was previously approved by the Ethical Committee of State University of Ponta Grossa (protocol number 6378/2016).

The animals were divided into four experimental groups, in order to obtain homogeneous groups regarding body mass and according to the administered formulations. Group 1 received cilostazol-loaded PCL-PEG nanocapsules at a dose of $50 \mathrm{mg} / \mathrm{kg} /$ day; Group 2 received PCL-PEG nanocapsules with no drug (unloaded nanocapsules); Group 3 received propylene glycol and water (70/30 v/v) (vehicle); and Group 4 received a suspension of cilostazol (pure cilostazol) in propylene glycol and water $(70 / 30, \mathrm{v} / \mathrm{v})$ at a dose of $50 \mathrm{mg} / \mathrm{kg} / \mathrm{day}$. The doses were administered by gastric gavage two times a day (07 and 19 hours), for a 30day period.

After twenty-four hours from the last dose administration, rats were sacrificed by anesthetic overdose with ketamine and xylazine $(75 \mathrm{mg} / \mathrm{kg}$ and $15 \mathrm{mg} / \mathrm{kg}$ intraperitoneally, respectively). Five milliliters of blood were collected by left ventricular puncture. Plasma was then stored in tubes without anticoagulant and immediately submitted to lipid profile analysis (triglycerides, total cholesterol and LDL-C and HDL-c fractions).

The abdominal aorta was surgically removed through a median longitudinal laparotomy, followed by divulsion and dissection by planes. Histological preparation included $10 \%(\mathrm{v} / \mathrm{v})$ formaldehyde storage, dehydration with increasing alcohol concentrations and paraffin inclusion to obtain 5-7 $\mu \mathrm{m}$ serial sections, which were stained with hematoxylin-eosin (HE).

The histological analysis was performed by a blinded examiner, using an optical microscope (Olympus BX 41) attached to a camera (Olympus DP72), under 400 times magnification. In each slide 5 different regions were analyzed, covering the three arterial tunics, with emphasis on the medial. The images were digitalized through the CellSens Standard $\AA$ program, obtaining the measurements in pixels, which were converted to micrometers.

A quantitative analysis of the presence of inflammatory response was performed through Image ${ }^{\circledR}$ Software (NIH, Bethesda, MD, USA) through the visualization of cell nuclei in the aortic artery tunica media, as well as the quantitative analysis to determine its thickness. Measurements to determine the thickness of the tunica media were performed at 10 points, perpendicular to the muscle fibers, with a 20 micrometer interval between them. The cellularity measurement was performed in an area of the tunica media equivalent to 800 micrometers, in each one of the slides.

Statistical analysis was done using SPSS $\AA$ program, Version 23.0. (IBM Corp., Armonk, NY, USA). After the evaluation of the normality of the data, one-way ANOVA test was used and Bonferroni multiple comparison test for post-hoc comparison. A $P<0.05$ was defined as statistically significant. The variables were presented by mean and standard deviation.

\section{RESULTS}

In Figure 1, we can observe the mean and the SD of the cellularity values. Although the group with unloaded nanocapsules (Group 2) presented the highest values (mean $=30.16$ cells) and the group with only propylene glycol (Group 3 ) the lowest (mean $=25.25$ cells), there was no statistically significant difference ( $p>0,05)$ among the treatments in this aspect.

In figure 2, the mean values of abdominal aortic artery thickness are observed. The unloaded nanocapsules group (Group 2) had the highest mean values (mean $=57.23 \mu \mathrm{m}$ ), while the group with nanoencapsulated cilostazol (Group 1) presented the lowest values (mean $=49.79 \mu \mathrm{m}$ ).

The one-way ANOVA test revealed a statistically significant difference among the groups $(p=0.043)$; however, when the Bonferroni multiple comparison test was applied, there was no significant difference between the groups.

The correlation between the variables was evaluated using Pearson's correlation test, which showed a slight positive correlation (0.333) between the variables aortic artery thickness and cellularity, but without statistical significance $(p=0.97)$.

Table 1 presents the mean values (SD) of Total Cholesterol, HDL-c, triglycerides, LDL-c in mg/dL. The one-way ANOVA test revealed statistical significance between the experimental groups for triglyceride $(p=$ $0.001)$ and HDL-c $(p=0.001)$. When applying the Bonferroni significance test for paired comparison, statistical differences were found between Group 1 and Group $2(p=0.002)$, and between Group 2 and Group $4(p=0.004)$ for triglyceride variable. Group 1 had the lowest mean value and Group 2 demonstrated the highest value for this biochemical parameter. Groups 1 and Group 2 also presented statistical difference ( $p$ 
$=0.001)$ for HDL-c variable, as well as Group 1 and Group $3(p=0.014)$. Concerning HDL-c, Group 1 showed the highest mean value while Group 2 had the lowest one.

No statistically significant differences were observed for the dependent variables total cholesterol and LDL-C among the evaluated groups.

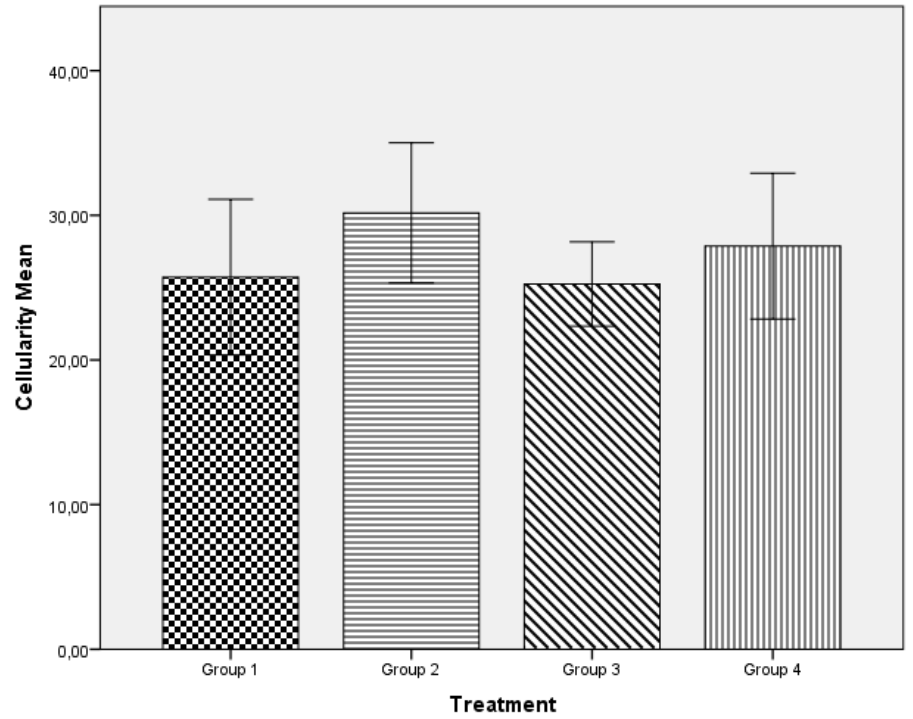

Figure 1. Cellularity Mean (SD) of the aorta artery for the experimental groups

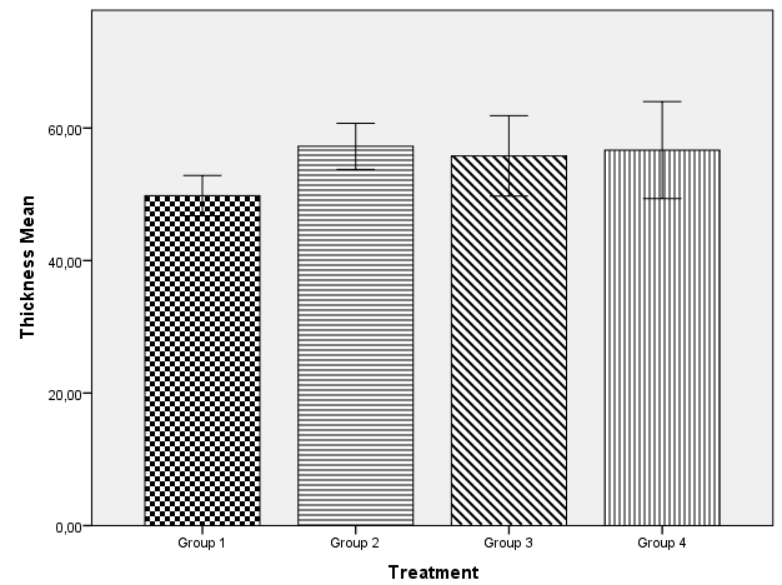

Figure 2. Thickness Mean (SD) of the aorta artery for the experimental groups

Table 1. Mean values (SD) of the lipid profile parameters for the experimental groups. Groups followed by distinct letters presented statistically significant differences. $(p<0,05)$.

\begin{tabular}{lllll}
\hline Group & Total cholesterol Mean (SD) & HDL-c Mean (SD) & Triglycerides Mean (SD) & LDL-c Mean (SD) \\
\hline $\mathbf{1}$ & $110.57(19.77) \mathrm{a}$ & $68.14(9.74) \mathrm{a}$ & $78.85(31,45) \mathrm{a}$ & $26.28(12.51) \mathrm{a}$ \\
\hline $\mathbf{2}$ & $114.75(11.96) \mathrm{a}$ & $49.45(6.91) \mathrm{b}$ & $171.75(49,43) \mathrm{b}$ & $40.12(15.16) \mathrm{a}$ \\
\hline $\mathbf{3}$ & $108.00(15.95) \mathrm{a}$ & $52.00(8.37) \mathrm{b}$ & $128.60(57,39) \mathrm{ab}$ & $34.60(15.24) \mathrm{a}$ \\
\hline $\mathbf{4}$ & $100.25(26.40) \mathrm{a}$ & $59.90(6.77) \mathrm{ab}$ & $88.12(33,95) \mathrm{a}$ & $31.50(24.04) \mathrm{a}$ \\
\hline
\end{tabular}

\section{DISCUSSION}

Cilostazol (CLZ) is a phosphodiesterase III inhibitor, characterized by its antiplatelet, antiproliferative and vasodilatory effects. It has demonstrated beneficial effects on the arteries, inhibiting the proliferation and growth of smooth muscle cells, increasing the release of nitric oxide by the endothelium and promoting 
angiogenesis [15-22], with limitations due to general side effects (headache, diarrhea, nausea) and its contraindication in heart failure [15,25]. In an attempt to improve pharmacotherapy, the use of the drugloaded nanocapsules may allow the minimization of side effects, a prolonged action and a reduction in the dosing frequency $[26,27]$.

However, there is still no wide references on the safety of nanocarriers and their toxicodynamic and toxicokinetic characteristics, as well as the efficacy of nanoencapsulated CLZ in relation to histological parameters, such as thickness and cellularity of the abdominal aorta and lipid profile. This lack of knowledge demands more scientific attention to investigate its efficacy in vascular surgery treatment.

In this sense, this work was designed to pre-clinically evaluate the effects of cilostazol-loaded nanocapsules (Group 1) on the morphological aspects of the aortic artery tunics of healthy rats and changes in the biochemical parameters of these animals in order to compare with the others groups of animals that received unloaded nanocapsules (Group 2), propylene glycol (Group 3) and pure cilostazol (Group 4).

Cilostazol is associated with decreased migration of smooth muscle cells into the endothelium, as well as its growth and proliferation [18-20]. In this context, it was expected that animals receiving the drug (Group 1 and Group 4) would present a smaller thickness of the aortic artery, since it is formed by smooth muscle cells. It was observed in the present study that the group treated with nanoencapsulated cilostazol (Group 1) presented the lowest mean thickness and the unloaded nanocapsules (Group 2) the highest; however, there was no statistical significance.

Regarding cellularity, there was no statistically significant difference in the amount of aortic artery cell nuclei among the groups evaluated. The groups that received cilostazol (Group 1 and Group 4) were expected to have the lowest mean values of cellularity, since the drug inhibits the proliferation and growth of muscle cells [18]. However, the group that received propylene glycol (Group 3) presented the lowest means, whereas the group with unloaded nanocapsules (Group 2) had the highest. In addition, Pearson's correlation test showed only a slight correlation, with no statistical significance between the variables thickness and cellularity.

The weak statistical significance in both the comparison of the cellularity means and the arterial thickness may be consistent with the age and conditions of the rats used (young rats, with no comorbidities nor hyperlipid diet) and the sample size. In addition, the short treatment time ( 1 month) may have contributed to the under-development of histological changes.

The use of cilostazol (Group 1, Group 4) promoted an improvement of the lipid profile when compared to the vehicle (Group 3), as found in the literature [23,24]. Likewise, the nanoencapsulated drug (Group 1) had positive responses in the lipid profile, with a statistically significant difference in relation to the unloaded nanocapsules (Group 2) and propylene glycol (Group 3) in the HDL-c parameter. Although the numerical results of the nanoencapsulated drug group (Group 1) were the most desirable, it should be noted that no statistical differences were observed between pure cilostazol (Group 4) and its nanoencapsulated form (Group 1). Perhaps a longer time of treatment or its application in older rats or with induced chronic diseases can discriminate the effects between the two groups.

Another relevant consideration is that the group in which unloaded nanocapsules were administered (Group 2) had the worst triglyceride values, statistically different from Group 1 and Group 4, treated with cilostazol. In this aspect, two issues must be highlighted. Firstly, the nanocapsules consist of an oily nucleus, wrapped in a polymeric shell [26], which may justify the increase in the triglycerides means in that group. Secondly, the nanocapsules containing cilostazol, due to the drug action mechanisms, avoided elevation of the mean value for this parameter, compensating the expected increase due to the presence of mediumchain triglycerides in the composition of this carrier system.

Regarding the mean serum total cholesterol and LDL-c, no statistical difference was found between the groups, which agrees with studies in the literature that reported that cilostazol does not affect these biochemical parameters [24].

Future studies may evaluate the use of cilostazol in rats on an hyperlipidic diet with atherosclerotic disease and more advanced age; as well as to carry out the treatment for a longer time. The understanding of the therapeutic properties and effectiveness of cilostazol associated with this new carrier system is crucial to verify its potential use in the PAOD treatment.

\section{CONCLUSION}

Polymeric nanocapsules containing cilostazol did not promote morphological changes in the cellularity and thickness of aortic artery arterial tunics of healthy and young Wistar rats. Concerning the lipid profile, this formulation statistically improved the HDL-C values, as well as the pure drug. Regarding the triglyceride values, cilostazol nanocapsules demonstrated a positive effect on this biochemical parameter. 


\section{REFERENCES}

1. Durazzo AES, et al. Doença arterial obstrutiva periférica: que atenção temos dispensado à abordagem clínica dos pacientes?. J Vasc Bras. 2005;4(3):255-64.

2. Diretrizes sobre Diagnóstico, Prevenção e Tratamento da Sociedade Brasileira de Angiologia e Cirurgia Vascular (SBACV) J Vasc Br. 2005;4(4):S222-8

3. Rafieian-Kopaei M, et al. Atherosclerosis: Process, Indicators, Risk Factors and New Hopes. Int J Prev Med 2014;5(8):927-46.

4. Smith C; Marks AD, Lieberman M. Bioquímica médica básica de Marks: uma abordagem clínica. Porto Alegre: Artmed, 2008.

5. Moise M, Kashyap V. Treatment of Aortoiliac Occlusive Disease: Medical versus Endovascular versus Surgical Therapy. Curr Treat Options Cardiovasc Med. 2011;13(2):114-28.

6. Neisen M. Endovascular Management of Aortoiliac Occlusive Disease. Seminars in Interventional Radiology. 2009;26(04):296-302.

7. Conte M, Pomposelli F, Clair D, Geraghty P, McKinsey J, Mills $\mathrm{J}$ et al. Society for Vascular Surgery practice guidelines for atherosclerotic occlusive disease of the lower extremities: Management of asymptomatic disease and claudication. J Vasc Surg. 2015;61(3):S2-41.e1.

8. Wooten C, Hayat M, du Plessis M, Cesmebasi A, Koesterer M, Daly K et al. Anatomical significance in aortoiliac occlusive disease. Clin Anat. 2014;27(8):1264-74.

9. Norgren L, Hiatt W, Dormandy J, Nehler M, Harris K, Fowkes F. Inter-Society Consensus for the Management of Peripheral Arterial Disease (TASC II). Eur J Vasc Endovasc Surg. 2007;33(1):pp.S1-75.

10. Ruggiero N, Jaff M. The Current Management of Aortic, Common lliac, and External Iliac Artery Disease: Basic Data Underlying Clinical Decision Making. Ann Vasc Surg. 2011;25(7):990-1003.

11. Regensteiner J, Ware J, McCarthy W, Zhang P, Forbes W, Heckman J et al. Effect of Cilostazol on Treadmill Walking, Community-Based Walking Ability, and Health-Related Quality of Life in Patients with Intermittent Claudication Due to Peripheral Arterial Disease: Meta-Analysis of Six Randomized Controlled Trials. J Am Geriatr Soc. 2002;50(12):1939-46.

12. Hobbs S, Marshall T, Fegan C, Adam D, Bradbury A. The effect of supervised exercise and cilostazol on coagulation and fibrinolysis in intermittent claudication: A randomized controlled trial. J Vasc Surg. 2007;45(1):65-70.

13.Treat-Jacobson D, Walsh M. 2016 ACC/AHA Guideline on the Management of Patients with Lower Extremity Peripheral Artery Disease: Process and Outcomes. J Vasc Nurs. 2017;35(2):116.

14. Thompson P, Zimet R, Forbes W, Zhang P. Meta-analysis of results from eight randomized, placebo-controlled trials on the effect of cilostazol on patients with intermittent claudication. Am. J. Cardiol. 2002;90(12):1314-19.

15.Pratt C. Analysis of the cilostazol safety database. Am. J. Cardiol. 2001;87(12):28-33.

16. Rosa M, Baroni G, Portal V. Cilostazol, um inibidor da fosfodiesterase III: perspectivas futuras na aterosclerose. Arq Bras Cardiol. 2006;87(5):e222-6.

17.Fukuoka T, Hayashi T, Hirayama M, Maruyama H, Tanahashi N. Cilostazol inhibits leukocyte-endothelial cell interactions in murine microvessels after transient bilateral common carotid artery occlusion. Brain Res. 2014;1543:173-8.

18.Kim M, Park K, Lee K, Kim H, Kim S, Kim C et al. Cilostazol Inhibits Vascular Smooth Muscle Cell Growth by Downregulation of the Transcription Factor E2F. Hypertension. 2005;45(4):552-6.

19. Chen W, Chen Y, Lin K, Hsuan Ting C, Yeh Y. Cilostazol Promotes Vascular Smooth Muscles Cell Differentiation Through the cAMP Response Element-Binding Protein-Dependent Pathway. Arterioscler Thromb Vasc Biol 2011;31(9):2106-13.

20. Hayashi S, Morishita R, Matsushita H, Nakagami H, Taniyama Y, Nakamura T et al. Cyclic AMP Inhibited Proliferation of Human Aortic Vascular Smooth Muscle Cells, Accompanied by Induction of p53 and p21. Hypertension. 2000;35(1):237-43.

21. Hashimoto A, Miyakoda G, Hirose Y, Mori T. Activation of endothelial nitric oxide synthase by cilostazol via a cAMP/protein kinase A- and phosphatidylinositol 3-kinase/Akt-dependent mechanism. Atherosclerosis 2006;189:350-7.

22.Ji JW, Mac Gabhann F, Popel AS. Skeletal muscle VEGF gradients in peripheral arterial disease: simulations of rest and exercise. Am J Physiol Heart Circ Physiol 2007;293:H3740-9.

23.Tani T, Uehara K, Sudo T, Marukawa K, Yasuda Y, Kimura Y. Cilostazol, a selective type III phosphodiesterase inhibitor, decreases triglyceride and increases HDL cholesterol levels by increasing lipoprotein lipase activity in rats. Atherosclerosis 2000;152:299-305.

24.Elam MB, Heckman J, Crouse JR, et al. Effect of the novel antiplatelet agent cilostazol on plasma lipoproteins in patients with intermittent claudication. Arterioscler Thromb Vasc Biol 1998;18:1942-7.

25. Cohn JN, Goldstein SO, Greenberg BH, Loreel BH, Bourge RC, Jaski BE, Gottlieb SO, McGrew F, DeMets DL, White BG, for the Vesnarinone Trial Investigators. A dose-dependent increase in mortality with vesnarinone among patients with severe heart failure. N Engl J Med 1998;339:1810-6.

26. Schaffazick SR, Guterres SS. Caracterização e estabilidade físico-química de sistemas poliméricos. Quim Nova. 2003;26(5):726-37.

27.Zampieri ALTC. Desenvolvimento, caracterização e avaliação da permeação cutânea da isoflavona genisteína em nanocápsulas poliméricas. Goiânia. Tese [Doutorado em Ciências da Saúde], Universidade Federal de Goiás; 2009. 
28.Gomes M, da Silva Nascimento N, Borsato D, Pretes A, Nadal J, Novatski A et al. Long-lasting anti-platelet activity of cilostazol from poly( $\varepsilon$-caprolactone)-poly(ethylene glycol) blend nanocapsules. Mater Sci Eng C. 2018;94:694702.

29. Masi C, Chandramohan C, Ahmed M. Immobilization of the Magnetic Nanoparticles with Alkaline Protease Enzyme Produced by Enterococcus hirae and Pseudomonas aeruginosa Isolated from Dairy Effluents. Braz. Arch. Biol. Technol. 2018;60(0).

30. Al-Rasheed N, Faddah L, Ibrahim H, Mohamed A, Al-Rasheed N, Abdelbaky N. Role of Carnosine and Melatonin in Ameliorating Cardiotoxicity of Titanium Dioxide Nanoparticles in the Rats. Braz. Arch. Biol. Technol. 2015;58(4):57786.

(c) 2020 by the authors. Submitted for possible open access publication under the terms and conditions of the Creative Commons Attribution (CC BY NC) license (http://creativecommons.org/licenses/by/4.0/). 